Supporting Information:

\title{
An All-Dielectric Polaritonic Metasurface with a Giant Nonlinear Optical Response
}

Raktim Sarma, ${ }^{1,2,+,}{ }^{*}$ Jiaming $\mathrm{Xu},{ }^{3,+}$ Domenico de Ceglia, ${ }^{4}$ Luca Carletti, ${ }^{4,5}$ Salvatore Campione, ${ }^{1}$ John Klem, ${ }^{1}$ Michael B. Sinclair, ${ }^{1}$ Mikhail A. Belkin,,${ }^{3,6}$ and Igal Brener ${ }^{1,2, *}$

${ }^{1}$ Sandia National Laboratories, Albuquerque, New Mexico 87123, USA

${ }^{2}$ Center for Integrated Nanotechnologies, Sandia National Laboratories, Albuquerque, New Mexico 87123, USA

${ }^{3}$ Department of Electrical and Computer Engineering, University of Texas at Austin, Austin, Texas 78712, USA

${ }^{4}$ Department of Information Engineering, University of Padova, Padua 35122, Italy

${ }^{5}$ Department of Information Engineering \& INO-CNR, University of Brescia, Brescia 25121 , Italy

${ }^{6}$ Walter Schottky Institut, Technische Universitat Munchen, Garching 85748, Bavaria, Germany

${ }^{+}$Equal contribution

*Email: (R. S.) rsarma@sandia.gov.*Email: (I.B.) ibrener@sandia.gov 


\section{S1. Numerical Simulations of Finite and Infinite Arrays of Dielectric Resonators}

As described in the main text, in our experiments the metasurface is illuminated with a finite spot size of radius $\sim 22 \mu \mathrm{m}$. Therefore, accurate predictions of the $\mathrm{SH}$ generation efficiencies require calculation of finite sized arrays. Fig. S1(a) shows numerically calculated maximum field enhancement for $E_{z}$ component $\left(\left|E_{z} / E_{\text {inc }}\right|\right)$ of arrays with different number of resonators $(N)$ with $R$ $=1.4 \mu \mathrm{m}(h=1.5 \mu \mathrm{m})$ as a function of pump wavelength. $N=1$ corresponds to an isolated metaatom and for $N>1(N=4,9,16,25,36)$, the meta-atoms were arranged in a $\sqrt{ } \mathrm{N} \times \sqrt{ } \mathrm{N}$ square lattice with period $(p)=3 R$. All these simulations include the contribution of the ISTs to the real and imaginary parts of the dielectric constant of the multi-QW layer. As shown in Fig. S1(a), for finite arrays, the field enhancement initially increases rapidly with $N$ and starts to converge for $N>25$. Interestingly, an infinite array overestimates the field enhancement: for any finite array the field enhancement is always smaller compared to an infinite array. For a given value of nonlinear susceptibility the field enhancement determines the second-harmonic generation (SHG) efficiency, and in Fig. S1(b), we can clearly see that the peak SHG efficiency (defined as the ratio of the maximum second-harmonic power to pump power) behaves similarly to the field enhancement with increasing $N$. As expected, the peak SHG efficiency is always higher for the case of an infinite array compared to finite array of resonators.

While the above discussion is relevant for determining the magnitude of SHG efficiency, it does not give information about the spectral dependence of the SHG efficiency. As described in the main text, to confirm the experimentally measured (Fig. 3(a) of main text) and numerically calculated (Fig. 3(b) of main text) spectral dependence of the SHG efficiency, we also calculated the effective nonlinear susceptibilities of an infinite metasurface with $R=1.4 \mu \mathrm{m}(h=1.5 \mu \mathrm{m}, p=$ 
$3 R$ ) as a function of pump wavelength. The effective nonlinear susceptibility of the metasurface is given by:

$$
\chi_{e f f,(l, m)}^{(2)}=\chi_{\mathrm{zzz}}^{(2)} \frac{\int_{0_{Q W}}^{v} E_{S H, z} E_{p, z} E_{p, z} d V}{\left|\vec{E}_{p, i}\right|^{2}\left|\vec{E}_{S H,(l, m)}\right| V_{Q W}}
$$

where, $\chi_{\mathrm{eff},(1, \mathrm{~m})}^{(2)}$ is the effective nonlinear susceptibility of the $(l, m)^{\mathrm{th}}$ free-space SHG diffraction order from the metasurface pumped at normal incidence (as mentioned in the main text, no SHG power is emitted in vertical direction), $\chi_{\mathrm{zzz}}^{(2)}$ is the intersubband nonlinear susceptibility coefficient of the multi-QWs shown in Fig. 1(c) of the main text, $\vec{E}_{p, i}$ is pump field amplitude incident normally from free space, $\vec{E}_{S H,(l, m)}$ is the SH field amplitude of the $(l, m)^{\text {th }}$ diffraction order of the metasurface in free space, $E_{S H, Z}$ and $E_{p, Z}$ are the z-components of the pump and SH fields in the cylindrical nanoresonator, and $V_{Q W}$ is the volume of the multi-QWs region in the nanoresonator. The overlap integrals for different values of $l$ and $m$ are evaluated as a function of pump wavelength. Since the effective nonlinear susceptibility of the metasurface includes the $\chi_{\mathrm{zzz}}^{(2)}$ of the multi-QWs, the field enhancements, and the nonlinear overlap factor, it can predict the overall spectral dependence of the SHG efficiency from the metasurface. Fig. S1(c), shows the calculations for the first two diffraction orders and the effective susceptibilities show a similar spectral dependence which further validates our experimental results and numerical simulations. 
(a)

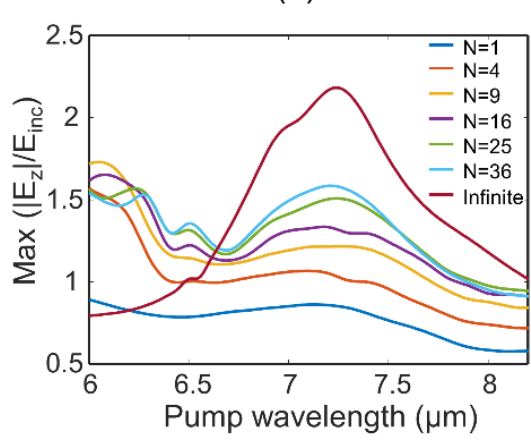

(b)

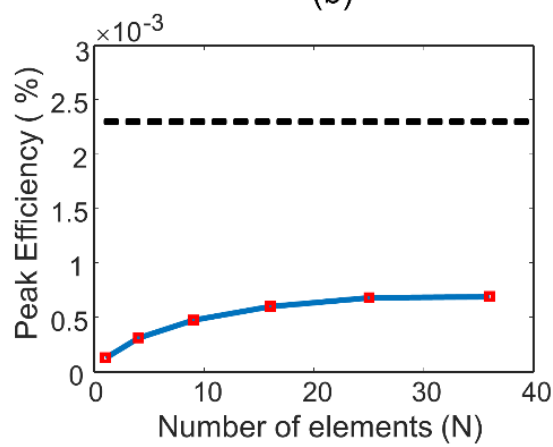

(c)

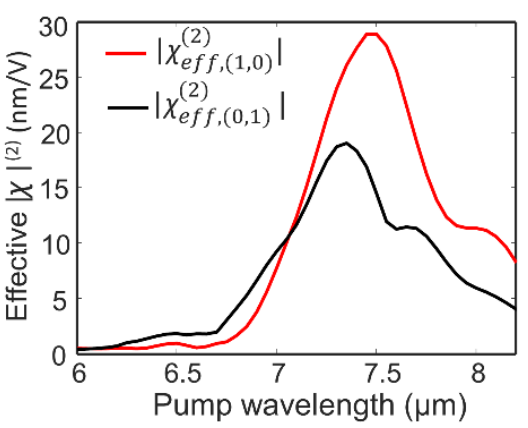

Figure S1. Linear and Nonlinear Numerical Simulations of Finite and Infinite Arrays. (a) Calculated maximum field enhancement for $\mathrm{E}_{\mathrm{z}}$ component $\left(\left|E_{z} / E_{\text {inc }}\right|\right)$ of arrays with different number of resonators $(N)$ with $R=1.4 \mu \mathrm{m}$ as a function of pump wavelength. The field enhancement initially increases rapidly with $N$ and starts to converge for $\mathrm{N}>$ 25. For any finite array, the field enhancement is always smaller than an infinite array. (b) Calculated peak SHG efficiency for arrays with different number of resonators $(N)$ with $R=1.4 \mu \mathrm{m}$. Similar to (a), the SHG efficiency first increases rapidly with $N$ and then converges for large values of $N$. The black dashed line corresponds to the peak SHG efficiency of an infinite array which is always higher than any finite sized array because of larger field enhancements. (c) Calculated effective nonlinear susceptibility $\chi_{e f f,(l, m)}^{(2)}$ of the first diffraction orders $(l=0, m=1$ and $l=1, m=0)$ of the metasurface with $R=1.4 \mu \mathrm{m}$.

\section{S2. Second-Harmonic Generation using Metasurfaces with Magnetic Quadrupole modes}

\section{coupled to the Intersubband Transitions.}

As described in the main text, the experimentally measured SHG efficiency for the metasurfaces with $R=1.4 \mu \mathrm{m}$ is lower than the actual SHG efficiency because of loss of SH signal due to diffraction. For a given numerical aperture (NA) of the collection optics and multi-QW design (which fixes the pump wavelength), there are two approaches that can be undertaken to minimize diffraction losses. The first approach is to fix the pump wavelength and increase the periodicity of the cylinders to decrease the diffraction angle. However, although this approach increases the collection efficiency, numerical simulations show a decrease in the SHG efficiency by more than an order of magnitude due to a lower field enhancement when the period $(p)$ increases from $3 R$ to $5 R$. The second approach, which we experimentally undertook, was to fix the pump wavelength and scale $R$ to a larger value together with the period (since $p=3 R$ ) such that the first order 
diffraction angle at the pump wavelength of $\sim 7.65 \mu \mathrm{m}$ is within the range of the numerical aperture (0.85) of the collection optics (for $R=2 \mu \mathrm{m}$ and pump wavelength $=7.65 \mu \mathrm{m}$, the diffraction angle for the first order is $\sim 39^{\circ}$ ). To pursue this approach, we fabricated a metasurface consisting of arrays of periodically spaced cylindrical dielectric resonators with $R=2 \mu \mathrm{m}$ and with the same multi-QW heterostructure. Since increasing $R$ also spectrally shifts the Mie modes to longer wavelengths, for these resonators the MD mode is shifted to longer wavelengths $(\sim 9 \mu \mathrm{m})$ and the magnetic quadrupole (MQ) mode now couples to the IST at $7.8 \mu \mathrm{m}$. Figs. S2(a, b) show the experimentally measured linear spectra for the case of $R=1.4 \mu \mathrm{m}$ (a) and $R=2 \mu \mathrm{m}$ (b) and the insets show the corresponding calculated far field patterns of SH for an isolated meta-atom $(N=$ 1). We can clearly see that for the case of $R=2 \mu \mathrm{m}$, the magnetic dipole (MD) mode is at a much longer wavelength $(\sim 9 \mu \mathrm{m})$ compared to $R=1.4 \mu \mathrm{m}$ and the MQ mode couples to the IST at $\sim$ $7.8 \mu \mathrm{m}$.

We note here that while the far field $\mathrm{SH}$ emission pattern for a single meta-atom (insets of Fig. S2(a) and (b)) are different for both the cases, what is important is that the for a finite array, the modified lobes of SH emission due to the periodicity and array factor are within the NA of the collection optics. Figs. S2(c, d) plot the far field SH emission pattern for a $6 \times 6$ array $(N=36)$ for $R=1.4 \mu \mathrm{m}$ (c) and $R=2 \mu \mathrm{m}$ (d). We can clearly see that for the case of $R=2 \mu \mathrm{m}$, all the dominant reflected lobes of SH emission are within the numerical aperture of the collection optics. Since for the case of $R=2 \mu \mathrm{m}$, we do not expect to lose signal due to diffraction, we expect the experimentally measured SHG efficiencies to be much larger than the case of $R=1.4 \mu \mathrm{m}$. Furthermore, since both MQ and MD resonances have similar $Q$ factors, we also expect that SHG efficiencies measured for $R=2 \mu \mathrm{m}$ should be comparable to the SH efficiency for $R=1.4 \mu \mathrm{m}$ 
when corrected for the collection efficiency. This is indeed what we observed experimentally and Fig. S3 shows the experimentally measured results. As shown in Fig. S3(a), the spectral

(a)

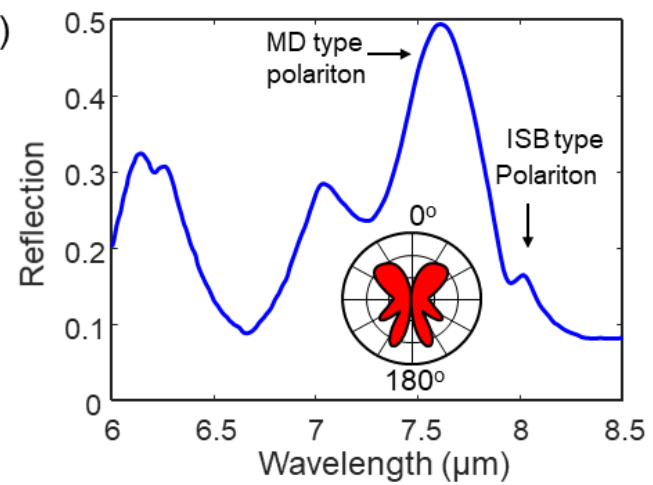

(c)

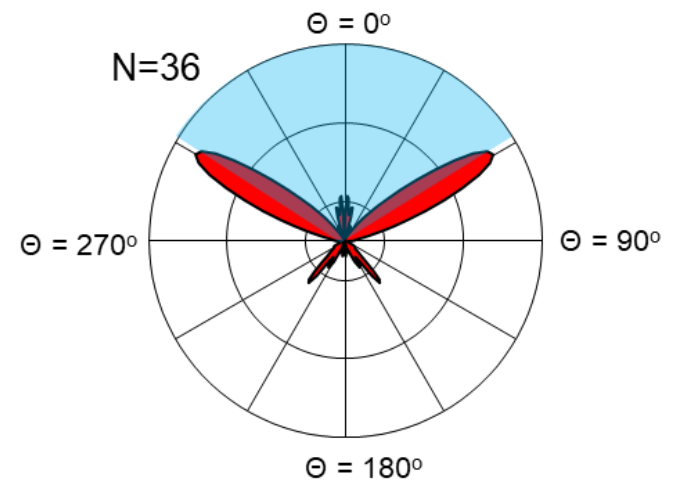

(b)

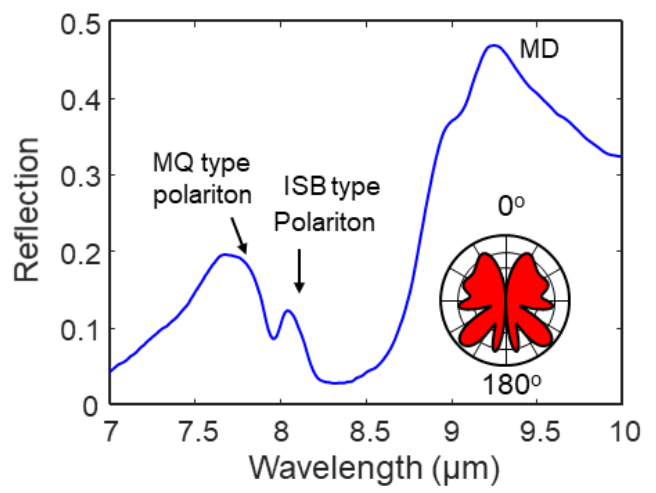

(d)

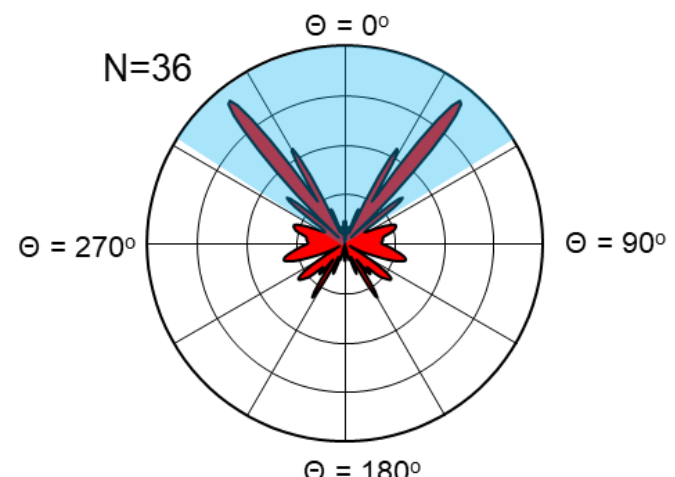

Figure S2. Linear reflectance measurements and nonlinear numerical simulation of metasurfaces with $R=1.4$ $\boldsymbol{\mu m}$ and $\boldsymbol{R}=\mathbf{2} \boldsymbol{\mu m}$. (a) Experimentally measured linear reflectance spectra of a metasurface with cylinders of $R=1.4$ $\mu \mathrm{m}$. Rabi splitting of the MD resonance can be seen indicating strong light-matter coupling between the MD resonance and the IST. The inset shows the calculated SH far field emission pattern for a single meta-atom at pump wavelength $=7.65 \mu \mathrm{m}$. (b) Experimentally measured linear reflectance spectra of a metasurface with cylinders of $R=2 \mu \mathrm{m}$. Rabi splitting of the MQ resonance can be seen indicating strong light-matter coupling between the MQ resonance and the IST. The inset shows the calculated SH far field emission pattern for a single meta-atom at pump wavelength $=7.65$ $\mu \mathrm{m}$. (c, d) Calculated far field emission pattern as a function of angle $(\theta)$ of SH in the $x-z$ plane from a $6 \times 6$ array $(N$ $=36$ ) with $R=1.4 \mu \mathrm{m}$ (c) and $R=2 \mu \mathrm{m}$ (d) at a pump wavelength of $7.65 \mu \mathrm{m}$ with pump intensity of $10 \mathrm{~kW} / \mathrm{cm}^{2}$. For $R=2 \mu \mathrm{m}$, the dominant reflected SH lobes are within the numerical aperture of the collection optics (shown by the shaded cone).

dependence of the SH conversion efficiency for the case of $R=2 \mu \mathrm{m}$ is very similar to $R=1.4 \mu \mathrm{m}$

(Fig. 3(a) of main text). Furthermore, the peak SH power conversion efficiency for $R=2 \mu \mathrm{m}$ is $~$ $0.015 \%$ (Fig. S3(b)) and the nonlinear conversion factor is $\sim 0.5 \mathrm{~mW} / \mathrm{W}^{2}$ (Fig. S3(c)) which are comparable to the conversion efficiencies for the case of $R=1.4 \mu \mathrm{m}$, when corrected for the collection efficiency (Fig. 4(a, b) of main text). This SH conversion efficiency is higher compared 
to record-high SHG power conversion efficiencies measured using GaAs/AlGaAs or GaP based all-dielectric metasurfaces [1-4].
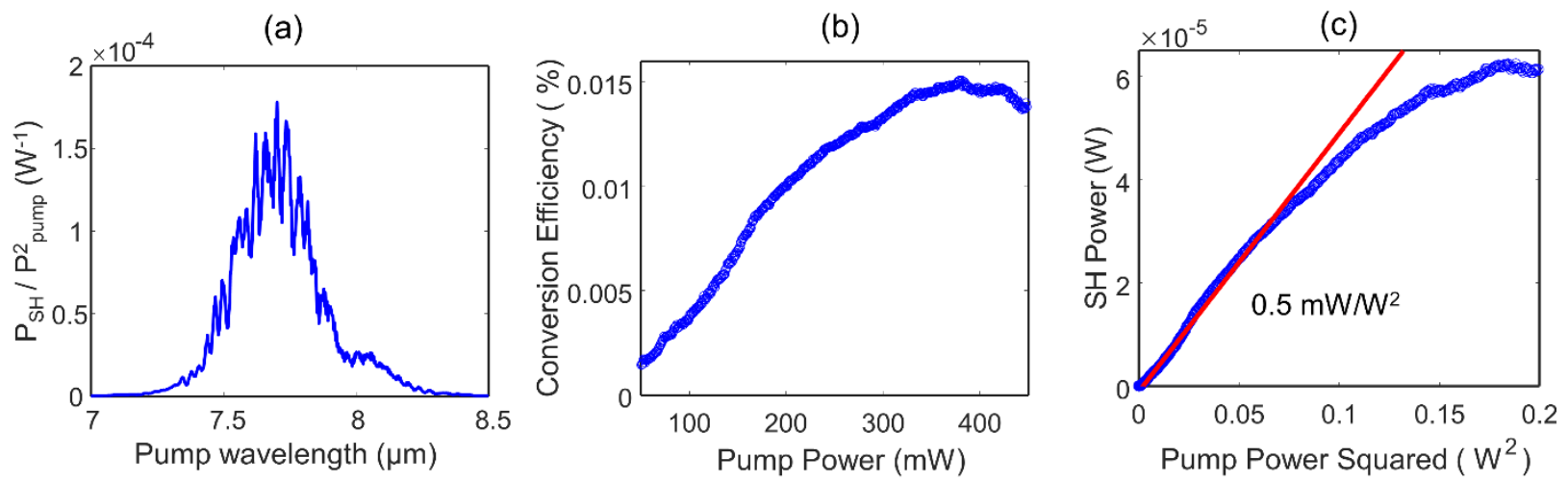

Figure S3. Experimental measurements of SHG from metasurfaces with MQ resonances coupled to ISTs. (a) Experimentally-measured reflected SH conversion efficiency in normalized units at normal incidence as a function of pump wavelength from a metasurface with cylinders of $R=2 \mu \mathrm{m}$. Two peaks at $\sim 7.6 \mu \mathrm{m}$ and $\sim 8 \mathrm{~m}$ are observed corresponding to the two polariton branches. The maximum SHG efficiency is observed at a pump wavelength of $\sim$ $7.65 \mu \mathrm{m}$ and the spectral dependence of SH conversion efficiency is very similar to the case of $R=1.4 \mu \mathrm{m}$ (Fig. 3(a) of main text). (b) Experimentally measured SH power conversion efficiency (defined as the ratio of SH power to pump power) as a function of incident peak pump power at a pump wavelength of $7.65 \mu \mathrm{m}$ of the metasurface with $R=2$ $\mu \mathrm{m}$. (c) Experimentally-measured peak SH power as function of square of incident peak pump power for the same metasurface and pump wavelength as in $(a, b)$. The slope of the linear fit (red line) determines the nonlinear conversion factor $\eta$ which is equal to $0.5 \mathrm{~mW} / \mathrm{W}^{2}$.

\section{S3. Details of Experimental and Simulation Methods}

\section{Metasurface Fabrication.}

The heterostructure used in this study was grown on a semi-insulating InP substrate using molecular beam epitaxy. It comprises multiple repetitions of the asymmetric coupled $\operatorname{In}_{0.53} \mathrm{Ga}_{0.47} \mathrm{As}$ QWs sandwiched between $\mathrm{Al}_{0.52} \mathrm{In}_{0.48} \mathrm{As}$ barrier layers as shown in Fig. 1(b). The total thickness of the multi-QW stack was $852 \mathrm{~nm}$. Since the minimum height of the resonator needs to be $\sim 1.5$ $\mu \mathrm{m}$ to support the lowest order $\mathrm{MD}$ mode, additional $\mathrm{Al}_{0.52} \operatorname{In}_{0.48} \mathrm{As}$ layers were grown symmetrically on top and bottom of the multi-QW stack to make the total epilayer thickness equal to $1.5 \mu \mathrm{m}$. The epilayer thickness determined the height $(h)$ of the resonators used in this study to be $1.5 \mu \mathrm{m}$. After growth, the wafer was flipped and adhesively bonded to a sapphire substrate 
using Benzocyclobutene. After bonding, the InP substrate was selectively removed to leave the epilayer on top of the sapphire substrate. The resonators were then defined via e-beam lithography. Hydrogen silsequioxane was used as the e-beam resist which was converted to $\mathrm{SiO}_{2}$ on e-beam exposure. After development, inductively-coupled plasma reactive ion etching was used for defining and fabricating the cylinders using the $\mathrm{SiO}_{2}$ layer on top of the cylinders as a mask. The $\mathrm{SiO}_{2}$ was not removed after the etching and together with the sapphire substrate on the bottom, it provided the refractive index contrast needed for the resonators to support the Mie modes. The Mie modes were spectrally scaled by changing the radius $(R)$ of the resonators. In all the metasurfaces, the periodicity ( $p$ ) of the array of the resonators along $x$ and $y$ was fixed to be $3 R$.

\section{Linear Numerical Simulations and Quantum Well Modeling.}

The linear reflectance spectra of the metasurfaces and electromagnetic field distributions inside the nanoresonators were calculated using a commercial finite-difference-time domain solver (Lumerical) as well as a frequency domain, finite-element solver (COMSOL). The multi-QW heterostructure embedded in the nanoresonators was modeled as a homogeneous, anisotropic layer with dielectric-constant tensor $\boldsymbol{\epsilon}_{\mathrm{QW}}=\epsilon_{t}(\widehat{\boldsymbol{x}} \hat{\boldsymbol{x}}+\widehat{\boldsymbol{y}} \widehat{\boldsymbol{y}})+\epsilon_{l} \widehat{\mathbf{z}} \hat{\boldsymbol{z}}$, where the transverse dielectric constant is $\epsilon_{t}=11.08$ and the longitudinal dielectric constant is

$$
\epsilon_{l}=11.08+\frac{f_{12} \omega_{12}^{2}}{\omega_{12}^{2}-\omega^{2}-2 i \omega \gamma_{12}}+\frac{f_{13} \omega_{13}^{2}}{\omega_{13}^{2}-\omega^{2}-2 i \omega \gamma_{13}} .
$$

The first Lorentzian term in Eq. (S2) models the first IST at $7.8 \mu \mathrm{m}\left(\omega_{12}=2 \pi \times 38.4 \times 10^{12} \mathrm{rad} / \mathrm{s}\right)$ and the second term models the second IST at the SH wavelength of $3.9 \mu \mathrm{m}\left(\omega_{13}=2 \pi \times 76.8 \times 10^{12} \mathrm{rad} / \mathrm{s}\right)$. The terms $f_{12}$ and $f_{13}$ are the oscillator strengths and are proportional to the product of the average doping density of the multi-QW heterostructure and the transition dipole length for the corresponding IST. The terms $\gamma_{12}$ and $\gamma_{13}$ represent the IST damping rate due to the resonant 
absorption and were determined by experimental absorption measurements. The transition dipole lengths, IST resonant frequencies, and band structures shown in Fig. 1(b) were determined from calculations using NextNano3, a commercial software by nextnano GmbH. The transition dipole lengths between the level $i$ and level $j$ for the optimized QW structure used in this study are $z_{12}=$ $1.6 \mathrm{~nm}, z_{23}=1.99 \mathrm{~nm}$ and $z_{31}=0.92 \mathrm{~nm}$. For all the electromagnetic simulations, the incident electric field was polarized along x direction. For the linear simulations shown in Fig. 2(b), an offnormal incident angle of $8^{\circ}$ was used to match the experimental conditions described below.

\section{Linear Optical Measurements of the multi-QWs and Metasurfaces.}

The normalized transmission spectrum of the semiconductor heterostructure shown in Fig. 2(a) was measured using Fourier-transform infrared spectroscopy (FTIR). As shown in the inset of Fig. 2(a), the bare wafer was polished to have 45 degrees input and output facets. The input direction (k) of the incident light used for the measurements is shown in the inset of Fig. 2(a). The IST resonant wavelength and linewidth were determined by taking the ratio of transmission of transverse magnetic polarized input light to transverse electric polarized input light. The normalized transmission spectrum shows a dip at the IST resonant wavelength which corresponds to a peak in absorption.

The reflectance from the fabricated metasurfaces (shown in Fig. 2(d)) was measured using an FTIR microscope fitted with a Schwarzschild objective of numerical aperture $=0.4$ which illuminated the samples at off-normal incidence angles $8^{\circ}<\Theta<23^{\circ}$. The incident light for the measurements was polarized along the $\mathrm{x}$-direction.

\section{Nonlinear Optical Measurements.}

For the nonlinear optical measurements, a tunable quantum cascade laser (QCL) was used as the pump and was operated at $250 \mathrm{kHz}$ repetition frequency with duty cycle of $10 \%$ and $1200 \mathrm{~mA}$ 
driving current. The nonlinear measurements were done at normal incidence where the sample was illuminated using an aspheric lens with numerical aperture of approximately 0.85 . The radius of the pump focal spot on the metasurface was measured by the knife-edge technique to be about $22 \mu \mathrm{m}$. The reflected SH light was collected using the same lens and was redirected either to an FTIR spectrometer to measure the spectra and or was focused onto a calibrated InSb detector using a 2-inch focal length parabolic mirror to measure the SH power.

\section{Nonlinear Numerical Simulations.}

Because of the IST selection rules, only out-of-plane polarized electric fields can couple to the ISTs and therefore the only non-zero element of the $\chi^{(2)}$-tensor in such a system is $\chi_{\mathrm{zzz}}^{(2)}$ which is given by the approximate expression

$$
\chi^{(2)}=\frac{e^{3}}{\epsilon_{0} \hbar^{2}} \frac{N_{d} z_{12} z_{23} z_{31}}{\left(\omega-\omega_{12}-i \gamma_{12}\right)\left(2 \omega-\omega_{13}-i \gamma_{13}\right)}
$$

where $e$ is the elementary charge, $\epsilon_{0}$ is the vacuum permittivity, $\hbar$ is the reduced Plank constant, $N_{d}$ is the average bulk doping density, $\gamma_{i j}$ represent the IST damping rate, and $z_{i j}$ are the transition dipole matrix lengths between levels $i$ and $j$. Figure 1(b) in the paper shows an 8-band k.p band structure calculation of the conduction band of a single period of the multi-QW system used in this study. The corresponding calculated $\chi_{\mathrm{zzz}}^{(2)}$, which is orders of magnitude larger than the bulk $\chi^{(2)}$ of standard III-V semiconductors, peaks at approximately $300 \mathrm{~nm} / \mathrm{V}$ for SHG at pump wavelength of $7.8 \mu \mathrm{m}$ and is shown in Fig. 1(c) of the paper.

Nonlinear numerical simulations (shown in Fig. 3) were performed using the frequency domain, finite-element method (COMSOL). The resonant, quadratic nonlinear susceptibility of the multi-

$\mathrm{QW}, \chi_{\mathrm{QW}}^{(2)}=\chi^{(2)} \hat{\mathbf{z}} \hat{\mathbf{z}} \hat{\mathbf{z}}$, was modeled based on Eq. S3. The calculations were done in the undepleted pump approximation and neglecting saturation effects of the ISTs. For simulating the nonlinear 
conversion factors of the metasurfaces, the electromagnetic problem at the pump frequency was solved in a unit cell using Floquet boundary conditions. Periodic port boundary conditions were used to launch the input pump as a plane wave polarized along the $\mathrm{x}$-direction at normal incidence to calculate reflection and transmission coefficients for all diffraction orders. The solution at the pump was then used to calculate the second-harmonic, nonlinear current source for the electromagnetic problem at the second-harmonic frequency. Similar to the electromagnetic problem at the pump frequency, Floquet boundary conditions and periodic ports were used in the unit cell simulations at the second-harmonic frequency, thereby enabling the possibility to extract conversion efficiencies for each diffraction order emitted in reflection and transmission. To calculate the SH conversion efficiencies, it was assumed that the entire SH power emitted in all the diffraction orders from the array was collected. For the isolated metaatom and the finite metasurfaces, a plane-wave excitation was considered at the pump frequency with a scattered field formulation of the problem, while perfectly-matched layers were introduced at the outer boundaries in order to absorb scattered light at the pump and SH frequency without reflections in the computational domain. The conversion efficiency was calculated by integrating the outwarddirected, time-averaged Poynting vector of the SH field over a hemi-sphere on the air side (reflected SH light), and considering as input pump power the nominal peak intensity in the experiment and an area equals to the geometrical cross section of the finite metasurface, i.e., $N p^{2}$, where $N$ is the total number of metaatoms in the array and $p$ the periodicity.

The effective $\chi^{(2)}$ elements shown here in Supplementary Information (Fig. S1(c)) were derived from overlap integrals in the meta-atoms, under simultaneous plane wave illumination of pump and SH signals in the linear regime, i.e., when the pump-SH nonlinear interaction is neglected. In particular, $\chi_{e f f,(1,0)}^{(2)}(l=1, m=0)$ is calculated when the pump signal is at normal 
incidence with $x$ polarized incident pump field $\vec{E}_{p, i}$, and the SH is obliquely incident at an angle $\sin \left(\theta_{S H}\right)=\frac{\lambda_{p}}{2 p} \quad\left(\lambda_{p}\right.$ is the pump wavelength; $p=$ is the nanoresonators spacing in the $\mathrm{x}-$ and $\mathrm{y}-$ direction) equal to the expected angle of emission for the $(1,0)$ diffraction order, with incident electric field $\vec{E}_{S H,(1,0)}$ polarized in the plane of incidence $(x, z)$. On the other hand, to calculate $\chi_{e f f,(0,1)}^{(2)},(l=0, m=1)$ we have considered the same illumination for the pump field and oblique incidence, at angles $\sin \left(\theta_{S H}\right)=\frac{\lambda_{p}}{2 p}$, with polarization of the incident $\vec{E}_{S H,(0,1)}$ field in the $(y, z)$ plane. The expressions for the effective $\chi^{(2)}$ are shown above where the overlap integrals are evaluated in the volume of the QW. We note that in the simulations, the pump beam is a normally incident plane wave.

\section{Origin of Saturation Effect of Nonlinearity.}

Unlike virtual transitions in bulk crystals, in this polaritonic system we rely on real electronic transitions to generate the nonlinearity. Since for SHG, the electrons in the ground state have to absorb a pump photon to get excited to a higher state, there is an effective decrease of electron population at the ground state leading to an effective decrease of the nonlinearity with increasing pump intensity, which eventually leads to a saturation effect.

\section{Effect of Off-Normal Incidence of Pump Light}

Our all-dielectric polaritonic metasurfaces can operate at both normal and off-normal incidence angles of the pump light. Shifting from normal to off-normal incidence will modify the mode profile of the MD mode. It will make the mode profile asymmetric with respect to the $\mathrm{x}$ and $\mathrm{y}$ axes and the out-of-plane $\left(\mathrm{E}_{\mathrm{z}}\right)$ components will increase as a function of incidence angle. Since only out-of-plane components couple to ISTs, compared to normal incidence, off-normal incidence will actually lead to even more efficient coupling of the pump light to the ISTs. 


\section{S4. Comparison of Different Approaches}

In the table below, we compare different metasurface-based approaches for second-harmonic generation. Since the efficiencies for a single meta-atom and a metasurface cannot be quantitatively compared, (discussed in the main text), in this table we have only included the works that involve metasurfaces.

Table S1. Comparison of different approaches for second-harmonic generation

\begin{tabular}{|c|c|c|c|c|c|c|}
\hline Category & Approach & $\begin{array}{l}\text { Material } \\
\text { System }\end{array}$ & $\begin{array}{l}\text { Operation } \\
\text { Wavelength }\end{array}$ & $\begin{array}{l}\text { Highest } \\
\text { Measured } \\
\text { Efficiency } \\
\frac{\boldsymbol{P}_{S H}}{\left(\boldsymbol{P}_{\text {pump }}\right)^{2}} \\
\left(\mathrm{~W} / \mathbf{W}^{2}\right)\end{array}$ & Advantages & Disadvantages \\
\hline $\begin{array}{l}\text { All- } \\
\text { Dielectric } \\
\text { Metasurface }\end{array}$ & $\begin{array}{l}\text { Low-order } \\
\text { Mie modes }\end{array}$ & $\begin{array}{l}\mathrm{AlGaAs} \\
{[2]}\end{array}$ & $>850 \mathrm{~nm}$ & $\begin{array}{l}1.5 \times 10^{-8} \\
\text { at } 1020 \mathrm{~nm} \text {. } \\
\end{array}$ & $\begin{array}{l}\text { - Low loss } \\
\text { - High damage } \\
\text { threshold } \\
\text { - Large mode } \\
\text { volumes. }\end{array}$ & $\begin{array}{l}\text { Moderate field } \\
\text { enhancements } \\
\text { - Moderate SHG } \\
\text { efficiencies } \\
\text { - Microscopic } \\
\text { control of material } \\
\text { nonlinearity not } \\
\text { possible. }\end{array}$ \\
\hline \multirow[t]{2}{*}{$\begin{array}{l}\text { All- } \\
\text { Dielectric } \\
\text { Metasurface }\end{array}$} & \multirow[t]{2}{*}{$\mathrm{BIC}$} & GaAs [3] & $>850 \mathrm{~nm}$ & $\begin{array}{l}4.5 \times 10^{-8} \\
\text { at } 1097 \mathrm{~nm} \text {. }\end{array}$ & $\begin{array}{l}\text { - Low loss } \\
\text { - Ultra-high } \\
\text { field } \\
\text { enhancement }\end{array}$ & \multirow{2}{*}{$\begin{array}{l}\text { - Very narrow } \\
\text { bandwidth } \\
\text { - Possibility of } \\
\text { photo damage, } \\
\text { nonlinear two- } \\
\text { photon absorption, } \\
\text { and free-carrier } \\
\text { absorption } \\
\text { - Microscopic } \\
\text { control of } \\
\text { nonlinearity not } \\
\text { possible } \\
\text { - May require } \\
\text { special pump } \\
\text { polarization } \\
\text { - May have } \\
\text { stringent }\end{array}$} \\
\hline & & $\mathrm{GaP}[4]$ & $>550 \mathrm{~nm}$ & $\begin{array}{l}5 \times 10^{-7} \\
\text { at } 1200 \mathrm{~nm} .\end{array}$ & - High SHG & \\
\hline
\end{tabular}




\begin{tabular}{|c|c|c|c|c|c|c|}
\hline \multirow[b]{2}{*}{$\begin{array}{l}\text { All- } \\
\text { Dielectric } \\
\text { Metasurface }\end{array}$} & & & & & & $\begin{array}{l}\text { requirements on } \\
\text { incidence angle. }\end{array}$ \\
\hline & $\begin{array}{l}\text { Leaky } \\
\text { mode } \\
\text { resonances } \\
\text { with ISTs }\end{array}$ & $\begin{array}{l}\text { InGaAs } \\
\& \\
\text { AlInAs } \\
{[5]}\end{array}$ & $\begin{array}{l}\text { Mid-IR }{ }^{+} \\
(>4 \mu \mathrm{m})\end{array}$ & $\begin{array}{l}\text { Low power } \\
1.1 \times 10^{-3} \\
\text { at } 9.5 \mu \mathrm{m} . \\
\text { High power } \\
3 \times 10^{-4} \\
\text { at } 9.5 \mu \mathrm{m} .\end{array}$ & $\begin{array}{l}\text { - Moderate to } \\
\text { large field } \\
\text { enhancements } \\
\text { - High SHG } \\
\text { efficiency } \\
\text { - Lower loss and } \\
\text { higher damage } \\
\text { threshold } \\
\text { compared to } \\
\text { IST-based } \\
\text { plasmonic } \\
\text { metasurfaces } \\
\text { - Broadband } \\
\text { SHG } \\
\text { - InGaAs/AlInA } \\
\text { s is lattice } \\
\text { matched to InP } \\
\text { substrate which } \\
\text { mitigates the } \\
\text { strain-induced } \\
\text { limitation on } \\
\text { the thickness } \\
\text { of the multi- } \\
\text { QW stack that } \\
\text { can be grown } \\
\text { - Large } \\
\text { transition } \\
\text { dipole } \\
\text { moments due } \\
\text { to small } \\
\text { electron } \\
\text { effective mass } \\
\text { in InGaAs } \\
\text { leading to large } \\
\text { magnitude of } \\
\text { the } \\
\text { nonlinearity. } \\
\text { - Ability to } \\
\text { design the } \\
\text { nonlinearity } \\
\text { via } \\
\text { intersubband } \\
\text { transitions } \\
\text { engineering. }\end{array}$ & $\begin{array}{l}\text { - Losses induced } \\
\text { by real electronic } \\
\text { transitions } \\
\text { - Saturation of } \\
\text { intersubband } \\
\text { nonlinearity at } \\
\text { high pumping } \\
\text { intensities } \\
\text { (specific values } \\
\text { are design } \\
\text { dependent). } \\
\text { - Operating } \\
\text { wavelengths are } \\
\text { limited to mid- } \\
\text { infrared spectral } \\
\text { range } \\
\text { - Works only at } \\
\text { off-normal } \\
\text { incidence angles. } \\
\text { - No possibility of } \\
\text { tailoring nonlinear } \\
\text { response at an } \\
\text { individual } \\
\text { nanoresonator } \\
\text { level. }\end{array}$ \\
\hline
\end{tabular}




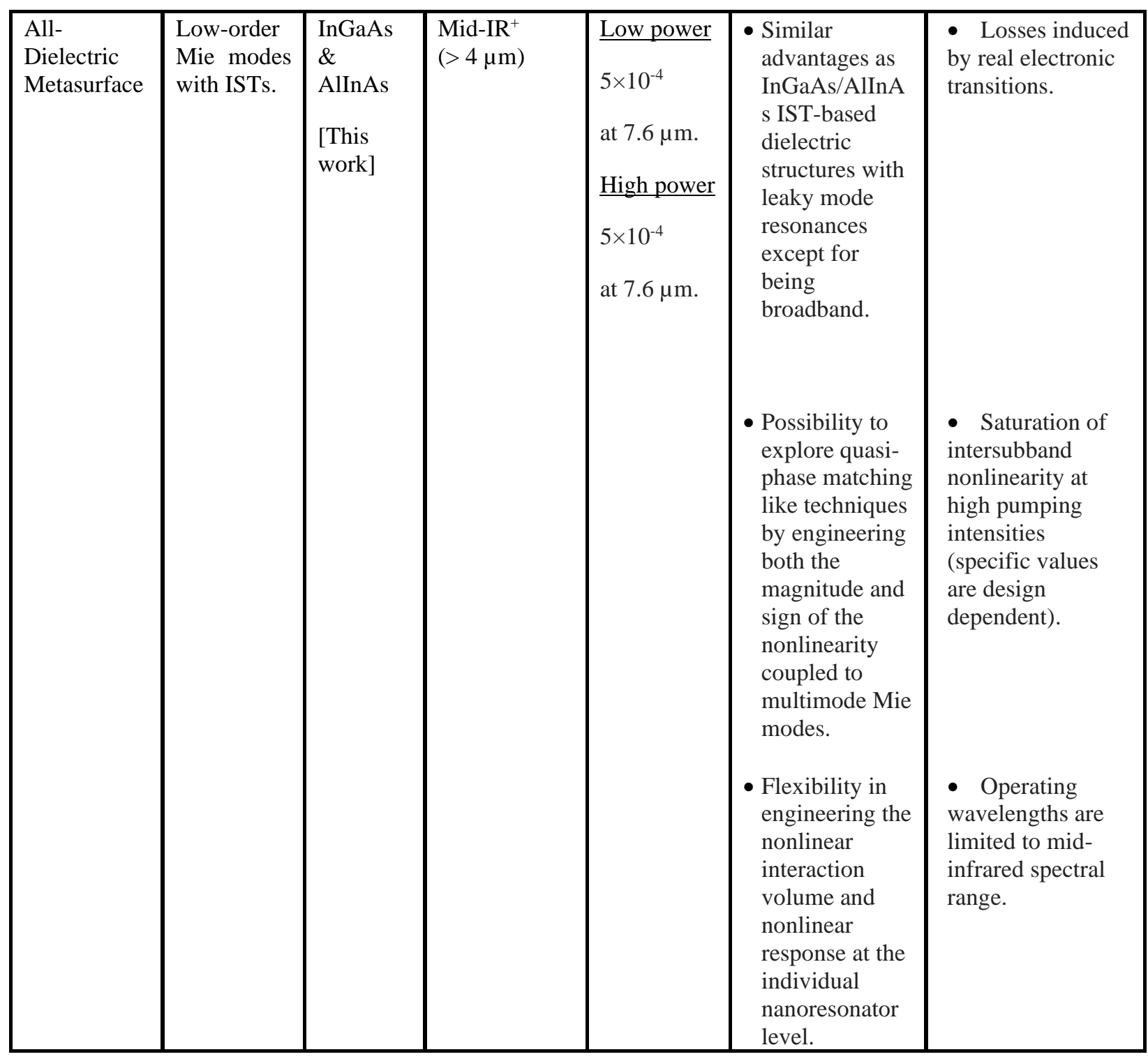




\begin{tabular}{|c|c|c|c|c|c|c|}
\hline $\begin{array}{l}\text { Plasmonic } \\
\text { Metasurface }\end{array}$ & $\begin{array}{l}\text { Plasmonic } \\
\text { meta-atoms } \\
\text { with ISTs } \\
\text { and metal } \\
\text { backplane }\end{array}$ & $\begin{array}{l}\text { InGaAs } \\
\& \\
\text { AlInAs } \\
{[6]}\end{array}$ & $\begin{array}{l}\text { Mid-IR }{ }^{+} \\
(>4 \mu \mathrm{m})\end{array}$ & $\begin{array}{l}\text { Low power } \\
16.8 \times 10^{-3} \\
\text { at } 9.9 \mu \mathrm{m} . \\
\text { High power } \\
5.3 \times 10^{-3} \\
\text { at } 9.9 \mu \mathrm{m} .\end{array}$ & $\begin{array}{l}\text { - Giant } \\
\text { nonlinearities } \\
\text { leading to } \\
\text { record-high } \\
\text { SHG } \\
\text { efficiencies. } \\
\text { - InGaAs/AlInA } \\
\text { s is lattice } \\
\text { matched to an } \\
\text { InP substrate } \\
\text { which } \\
\text { mitigates the } \\
\text { strain-induced } \\
\text { limitation on } \\
\text { the thickness } \\
\text { of the multi- } \\
\text { QW stack that } \\
\text { can be grown } \\
\text { - Large } \\
\text { transition } \\
\text { dipole } \\
\text { moments due } \\
\text { to small } \\
\text { electron } \\
\text { effective mass } \\
\text { in InGaAs lead } \\
\text { to large } \\
\text { magnitude of } \\
\text { the } \\
\text { nonlinearity } \\
\text { with the ability } \\
\text { to design the } \\
\text { nonlinearity. }\end{array}$ & $\begin{array}{l}\text { - Losses and low } \\
\text { damage thresholds } \\
\text { due to the } \\
\text { presence of } \\
\text { metals. } \\
\text { - Losses induced } \\
\text { by real electronic } \\
\text { transitions. } \\
\\
\text { - Saturation of } \\
\text { intersubband } \\
\text { nonlinearity at } \\
\text { high pumping } \\
\text { intensities } \\
\text { (specific values } \\
\text { are design } \\
\text { dependent). } \\
\text { - Operating } \\
\text { wavelengths are } \\
\text { limited to mid- } \\
\text { infrared spectral } \\
\text { range. }\end{array}$ \\
\hline
\end{tabular}

+ Note: The wavelength of operation for IST based structures can be tuned to shorter near-IR wavelengths by choosing different material systems such as InAs/AlSb or III-Nitrides.

\section{S5: Optimization of Second-Harmonic Generation using All-Dielectric Intersubband Polaritonic Metasurface}

The results presented in this work constitute a proof-of-concept demonstration and there is room for much further improvement of SHG efficiencies using these IST-based all-dielectric polaritonic metasurfaces. To maximize the second-harmonic generation efficiency, we must simultaneously optimize 6 quantities: the field enhancement at the pump frequency, the field enhancement at the 
second harmonic frequency, the overlap between the modes at the pump and second harmonic frequencies, the emission directionality, the magnitude of the nonlinearity, and saturation intensity of the intersubband transition. While the field enhancements, mode overlaps, and emission directionalities can be optimized using electromagnetic mode engineering, optimization of the magnitude of nonlinearity and saturation intensity requires exploration of new types of quantum heterostructures. For example, one approach to performing the electromagnetic mode engineering would be to use topology optimization along with gradient based optimization techniques to design meta-atoms of shapes which are not possible to predict using pure physical intuition [7]. Such optimization techniques can be used to design meta-atoms which can have large field enhancements (similar to BICs $[3,4,8,9]$ ) and good mode overlaps. The same optimization technique can also be used to optimize the emission directionality by designing periodic arrays of these meta-atoms such that most of the SH signal is radiated along the normal direction or zeroth diffraction order. This will mitigate the problem of large diffraction angles in the reflection geometry and the issue of total internal reflection of the transmitted SH signal (which significantly reduces the collection efficiency) while maintaining high SHG efficiency. Finally, to optimize the magnitude of the nonlinearity and to increase the saturation intensity, asymmetric multi-QW structures with a single intersubband transition that is resonant with the SH frequency can be explored [10]. Such a design which uses a combination of real and virtual transitions to generate SH signal will not have any absorption at the pump frequency and will allow us to maximize $\left|\chi_{\mathrm{zzz}}^{(2)} f_{p}^{2}\right|^{2}$ where $f_{\mathrm{p}}$, is the field enhancement $\left(f_{p}=\left|E_{z} / E_{\text {incident }}\right|\right.$ where $E_{\text {inc }}$ is the amplitude of the electric field in the incident wave) of the $E_{z}$ field component at the pump wavelength. Furthermore, because of the absence of a real electronic transition at the pump frequency, the issue 
of saturation will also be significantly mitigated. Such an optimization is beyond the scope of this work and will be presented in a future study.

\section{REFERENCES}

(1) Liu, S.; Sinclair, M. B.; Saravi, S.; Keeler, G. A.; Yang, Y.; Reno, J.; Peake, G. M.; Setzpfandt, F.; Staude, I.; Pertsch, T.; Brener, I. Resonantly Enhanced Second-Harmonic Generation Using III-V Semiconductor All-Dielectric Metasurfaces. Nano Lett. 2016, 16(9), 5426-5432.

(2) Marino, G.; Gigli, C.; Rocco, D.; Lemaitre, A.; Favero, I.; Angelis, C. D.; Leo, G., ZeroOrder Second Harmonic Generation from AlGaAs-on-Insulator Metasurfaces. ACS Photonics 2019, 6(5), 1226-1231.

(3) Vabishchevich, P. P.; Liu, S.; Sinclair, M. B.; Keeler, G. A.; Peake, G. M.; Brener, I.; Enhanced Second-Harmonic Generation Using Broken Symmetry III-V Semiconductor Fano Metasurfaces. ACS Photonics 2018, 5(5), 1685-1690.

(4) Anthur, A. P.; Zhang, H.; Dominguez, R. P.; Kalashnikov, D. A.; Ha, S. T.; MaB, T. W. W.; Kuznetsov, A. I.; Krivitsky, L. Continuous Wave Second Harmonic Generation Enabled by Quasi-Bound-States in the Continuum on Gallium Phosphide Metasurfaces. Nano Lett. 2020, 20(12), 8745-8751.

(5) Sarma, R.; de Ceglia, D.; Nookala, N.; Vincenti, M. A.; Campione, S.; Wolf, O.; Scalora, M.; Sinclair, M. B.; Belkin, M. A.; Brener, I. Broadband and Efficient Second-Harmonic Generation from a Hybrid Dielectric Metasurface/Semiconductor Quantum-Well Structure. ACS Photonics 2019, 6(6), 1458-1465.

(6) Lee, J.; Nookala, N.; Gomez-Diaz, J. S.; Tymchenko, M.; Demmerle, F.; Boehm, G.; Amann, M. C.; Alu, A; Belkin, M. A. Ultrathin Second-Harmonic Metasurfaces with Record-High Nonlinear Optical Response. Adv. Optical Mater. 2016, 4 (5), 664-670.

(7) Lin, Z.; Liang, X.; Loncar, M.; Johnson, S. G.; Rodriguez., A. W. Cavity-Enhanced SecondHarmonic Generation via Nonlinear-Overlap Optimization. Optica 2016, 3 (3), 233-238.

(8) Koshelev, K.; Kruk, S.; Gaykazyan, E. M.; Choi, J. H.; Bogdanov, A.; Park, H. G.; Kivshar, Y. Subwavelength Dielectric Resonators for Nonlinear Nanophotonics. Science 2020, 367(6475), 288-292.

(9) Zhou, Y.; Zheng, H.; Kravchenko, I. I.; Valentine, J. Flat Optics for Image Differentiation. Nat. Photonics 2020, 14, 316-323.

(10) Rosencher, E.; Fiore, A.; Vinter, B.; Berger, V.; Bois, Ph.; Nagle, J. Quantum Engineering of Optical Nonlinearities. Science 1996, 271, 168-173. 